\title{
Ist die Ablehnung von invasiver oder noninvasiver Beatmung bei einem Kind mit spinaler Muskelatrophie aus ethischer Sicht vertretbar?
}

\author{
Martina Radner (D) - Annette Henry
}

Eingegangen: 23. Mai 2017 / Angenommen: 25. Januar 2018 / Online publiziert: 15. Februar 2018

(c) Der/die Autor(en) 2018. Dieser Artikel ist eine Open-Access-Publikation.

Zusammenfassung Wir berichten von einem Säugling, bei dem im Alter von 7 Wochen eine spinale Muskelatrophie Typ 1 (Werdnig-Hoffmann) diagnostiziert wurde. In einem ausführlichen Gespräch mit den behandelnden Ärzten entschieden sich die Eltern gegen eine maschinelle Beatmung im Falle einer akuten oder chronischen respiratorischen Insuffizienz des Kindes. Bei der häuslichen Betreuung wurden alle anderen palliativen Maßnahmen zur Optimierung der Lebensqualität des Kindes wahrgenommen. Der Knabe verstarb im Alter von 7 Monaten zu Hause. Ob die maschinelle Dauerbeatmung eines Kindes mit SMA 1 individuelle Entscheidung der Eltern sein sollte, oder von ärztlicher Seite generell nicht angeboten werden soll, wird in der aktuellen medizinischen Literatur durchaus kontroversiell diskutiert

Schlüsselwörter Spinale Muskelatrophie Typ 1 . Werdnig Hoffmann · Invasive Beatmung • Ethik • Lebensqualität

Is the refusal of invasive or noninvasive ventilation in a child with spinal muscular atrophy ethically justified?

Summary We report about an infant who was diagnosed with spinal muscular atrophy type 1 (WerdnigHoffmann) at the age of 7 weeks. In a detailed discussion with the physicians the parents decided against mechanical ventilation in the event of either acute or

Dr. M. Radner $(\bowtie) \cdot$ Dr. A. Henry, MSC

Wiens Mobiles Kinderhospiz MOMO,

Schulgasse 43, 1180 Wien, Österreich

martina.radner@kinderhospizmomo.at

Dr. A. Henry, MSC

annette.henry@kinderhospizmomo.at chronical respiratory failure. During care at home all palliative actions were taken to optimize the quality of life for the child. The boy died at the age of 7 months at home. Whether continuous mechanical ventilation in a child with spinal muscular atrophy type 1 should be the individual decision of the parents or whether it should never be offered by the physicians is discussed controversially in the present medical literature.

Keywords Spinal muscular atrophy type 1 . Werdnig Hoffmann · Mechanical ventilation · Ethics · Quality of life

\section{Einleitung}

Spinale Muskelatrophie Typ 1 (SMA 1) ist eine autosomal rezessiv vererbte, progredient verlaufende Erkrankung, die durch muskuläre Hypotonie und zunehmende respiratorische Insuffizienz gekennzeichnet ist [1]. Ohne maschinelle Beatmung verstirbt ein Großteil der Betroffenen im ersten oder zweiten Lebensjahr.

Daher ist das Anlegen eines Tracheostomas mit konsekutiver maschineller Beatmung eine wichtige Option zur Lebensverlängerung dieser Patienten.

In der Literatur ist jedoch die ethische Rechtfertigung dieser Maßnahme sehr umstritten, da die Lebensqualität dieser Patienten sehr unterschiedlich beurteilt wird. Einerseits sind Kinder mit spinaler Muskelatrophie sehr wach und aufmerksam, da sie intellektuell nicht beeinträchtigt sind, andererseits bestehen durch die muskuläre Hypotonie keine oder nur äußerst eingeschränkte Möglichkeiten zur Kommunikation mit der Umwelt.

Daraus ergibt sich das ethische Dilemma, ob eine Lebensverlängerung durch maschinelle Beatmung eventuell mit einer deutlichen Verschlechterung der 
Lebensqualität beziehungsweise der Verlängerung eines unzumutbaren Leidens einhergehen könnte.

\section{Fallbericht}

Laurenz wurde als erstes Kind gesunder Eltern per sectionem aus mütterlicher Indikation am Termin geboren. Im Alter von 4 Wochen wurde im Rahmen der Mutter-Kind-Pass-Untersuchung eine muskuläre Hypotonie und verminderte Spontanmotorik festgestellt. Der behandelnde Kinderarzt vermutete eine muskuläre Erkrankung und überwies das Kind an eine Kinderabteilung zur weiteren Abklärung.

Im Rahmen der weiteren Untersuchungen zeigte sich eine erhöhte Kreatinkinase (237U/1). Es erfolgte eine genetische Abklärung unter der Verdachtsdiagnose einer spinalen Muskelatrophie. Diese ergab eine 5qassoziierte Deletion des Exons 7 und 8 und somit das Vorliegen eines SMA 1 (spinale Muskelatrophie Typ I) - Gens.

Im Alter von 2 Monaten wurde mit regelmäßiger ambulanter Physiotherapie begonnen, einen Monat später nahmen die Eltern Kontakt mit dem mobilen Kinderhospiz auf.

Im Alter von 4 Monaten unterschrieben die Eltern im Rahmen eines stationären Aufenthalts wegen eines respiratorischen Infekts nach einem ausführlichen Gespräch mit den behandelnden Ärzten ein Therapiezielprotokoll, in dem festgehalten wurde, dass keine maschinelle Beatmung und bei Herz-Kreislaufstillstand keine Reanimation erfolgen sollen.

$\mathrm{Ab}$ diesem Zeitpunkt erfolgten regelmäßige Besuche der Mitarbeiterinnen des mobilen Kinderhospizes MOMO sowie einer Physiotherapeutin. Im 7. Lebensmonat konnte mit oraler Ernährung keine adäquate Gewichtszunahme mehr gewährleistet werden, da der Knabe unter zunehmenden Schluckproblemen litt. Es wurde eine Nasogastralsonde gelegt und die orale Ernährung wegen zunehmender Aspirationsgefahr beendet.

Kurz darauf wurde eine kontinuierliche Sauerstoffzufuhr begonnen und ebenfalls regelmäßig durch die Eltern Schleim aus dem Nasen-Rachenraum abgesaugt.

Da es in der Folge immer wieder zu Episoden mit Blässe und Unruhe kam, wurde unter der Annahme, dass diese durch Schmerzen ausgelöst seien, eine regelmäßige Therapie mit Metamizol eingeleitet.

Kurz nach Vollendung des achten Lebensmonats verstarb Laurenz zu Hause.

\section{Psychosoziale Situation der Familie}

Nach der ersten Schockphase zum Zeitpunkt der Diagnosestellung waren die Eltern sehr gefasst und entschieden in jeder neuen Phase der Erkrankung übereinstimmend für oder gegen die jeweils vom medizinischen Personal vorgeschlagene Maßnahme. Sie begründeten ihre Entscheidungen stets mit ihrer
Sorge um das subjektive Wohlbefinden ihres Sohnes. Mit diesem Argument entschieden sie auch im Rahmen der Erstellung eines Therapiezielprotokolls gegen eine manuelle Reanimation mit Herzdruckmassage und gegen noninvasive und invasive Beatmung. Sie nahmen jedoch viele Belastungen für sich selbst in Kauf (Sauerstoffversorgung und Absaugen zu Hause, sehr unruhige Nächte), um ihrem Sohn trotz seiner Erkrankung eine größtmögliche Lebensqualität zu erhalten.

Es bestand auch großer emotionaler Rückhalt durch die Eltern der Mutter, die zwar verständlicherweise sehr betroffen von Diagnose und Prognose ihres Enkelkindes waren, jedoch immer wieder zu Besuch kamen oder zu Besuchen einluden. Eine enorme Ressource bestand in der Beziehung von Mutter und Vater des Kindes zueinander. Sie versuchten, wann immer es der Zustand des Kindes zuließ, miteinander zusammen mit dem Kind spazieren oder Essen zu gehen und damit ein „normales“ Leben zu führen.

Die Mutter nahm über Internetforen Kontakt mit anderen betroffenen Eltern auf und informierte sich umfassend über den $\mathrm{zu}$ erwartenden Verlauf der Krankheit. Bemerkenswert war auch, dass sich die Eltern intensiv mit der Frage der Organspende ihres Kindes auseinandersetzten, um dem Leben und Leiden ihres Kindes „einen Sinn zu geben“. Die Organspende konnte jedoch aufgrund der genetischen Determination der Erkrankung nicht in Erwägung gezogen werden.

Aufgrund der vielfältigen eigenen Ressourcen nahmen die Eltern auch erst sehr spät im Krankheitsverlauf (im letzten Lebensmonat des Kindes) die Unterstützung durch die Psychologin des Kinderhospizes MOMO in Anspruch.

\section{Fragestellung}

Ist das Anlegen eines Tracheostomas und die Einleitung der maschinellen Beatmung bei einem Kind mit SMA 1 ethisch gerechtfertigt oder wird damit das Leiden eines Menschen mit sehr geringer Lebensqualität in unzumutbarer Weise verlängert?

\section{Diskussion}

Obwohl die Option einer maschinellen Beatmung eines Kindes mit SMA 1 in der vorliegenden Literatur vorwiegend negativ beurteilt wird, gibt es durchaus auch Arbeitsgruppen, die der maschinellen Beatmung positiv gegenüberstehen.

Ein australisches Team um Kelly Gray berichtet vom Tagesablauf eines beatmeten achtjährigen Mädchens mit SMA 1. Das Mädchen lebt in einer Kinderklinik, da die Eltern sich außerstande sahen, ihr beatmetes Kind zu Hause zu betreuen. Sie leidet aufgrund ihrer Erkrankung zusätzlich an einer schweren Osteopenie, sowie chronischen Schmerzen infolge massiver Kontrakturen. Sie kann mit der Umwelt nur wenig kom- 
munizieren, kann aber lächeln und ihre Stimmung kann aus physiologischen Zeichen (Herz- und Atemfrequenz) interpretiert werden.

Die Gruppe kommt in der Diskussion des vorliegenden Falles zur Konklusion, dass die Langzeitbeatmung und damit Lebensverlängerung dieses Kindes nicht gerechtfertigt sei. Man müsse davon ausgehen, dass die Lebensverlängerung für das Mädchen in diesem Fall eine unzumutbare Belastung darstelle und damit die Nachteile die Vorteile der Lebenserhaltung überwiegen [2].

$\mathrm{Zu}$ einer ähnlichen Erkenntnis kommt ein Schweizer Team, das die Problematik der Entscheidung durch Einsetzen eines multiprofessionellen Teams zu lösen versucht. Dabei werden alle in den Fall involvierten und interessierten Fachpersonen zu einer ethischen Fallbesprechung (EFB) einberufen. Von einer Teilnahme der betroffenen Eltern wurde Abstand genommen, da die Diskussionen und Formulierungen eventuell für diese zu anspruchsvoll sein konnten.

Im Rahmen dieser EFB wurde zunächst das ethische Dilemma der Langzeitbeatmung bei Vorliegen einer rasch progredienten Erkrankung formuliert. Anschließend wurde eine Kontextanalyse vorgenommen, die die in diesem Falle nicht existenten kurativen Ansätze der Erkrankung, den Zustand des Kindes und die Einstellung der Eltern festhielt. Nun wurde eine Werteanalyse vorgenommen, die sich in erster Linie auf das ethische Prinzip Gutes zu tun und Leiden zu vermeiden stützte. Im Anschluss erfolgte das Erarbeiten der Entscheidungsoptionen und deren Analyse. Schließlich kam das Team in diesem Fall zu dem Konsens, von einer belastenden, invasiven Therapie Abstand zu nehmen. Die Eltern konnten sich schließlich diesem Konsens anschließen und die geplante Vorgehensweise akzeptieren [3].

Eine Arbeitsgruppe aus Salt Lake City analysierte retrospektiv 49 Patienten mit SMA 1, von denen 26 beatmet und 23 palliativ betreut wurden. Im Alter von 4 Jahren waren von den beatmeten Kinder noch $50 \%$ und von den nicht beatmeten $12 \%$ am Leben.

Damit kamen die Autoren zur Erkenntnis, dass die Langzeitbeatmung definitiv einen Vorteil in sich birgt, was die Lebenserwartung betrifft. Es wird aber auch darauf hingewiesen dass die Langzeitbeatmung auch eine deutliche Belastung der betroffenen Familien darstellt, auf die bereits beim Aufklärungsgespräch vor der Entscheidungsfindung der Eltern spezielles Augenmerk gelegt werden muss [4].

Eine weitere australische Gruppe berichtet von einem 18 Monate alten Kind mit SMA 1, das im Alter von 12 Wochen aufgrund eines Infektes respiratorisch insuffizient wurde und in der Folge in einem Krankenhaus, in dem die Diagnose des Kindes nicht bekannt war, intubiert wurde. Da die Eltern in eine Beendigung der Beatmungstherapie nicht einwilligten, war das Kind bis zum Zeitpunkt des Berichts (12 Monate später) noch immer an der Intensivstation stationär. In der vorliegenden Arbeit nehmen die einzelnen be- teiligten Ärzte sowie eine Ethikerin zu dem Fall Stellung.

Es wird zu bedenken gegeben, dass die Entscheidung zur Beendigung dieser Therapie weit schwieriger ist, als die Entscheidung über die primäre Intubation. Daher meint der Autor, dass diesen Patienten gar keine Beatmungstherapie angeboten werden sollte. Ein weiterer behandelnder Arzt meint, dass die Belastung durch das Fortschreiten der Krankheit jedenfalls unzumutbar sei, und daher alles daran gesetzt werden müsse, die Eltern von dieser Tatsache zu überzeugen. Falls dies nicht möglich sei, solle die Entscheidung über die Fortsetzung der Therapie einem Gericht überlassen werden. In einer weiteren Stellungnahme wird vorgeschlagen, dass im Rahmen einer Gesetzgebung festgelegt werden sollte, Kinder mit der Diagnose einer SMA 1 nicht zu beatmen. Er zitiert aber einen britischen Gerichtsentscheid, in dem die Beendigung der Beatmung eines Kindes mit SMA 1 mit der Begründung abgelehnt wurde, dass es nicht im besten Interesse des Kindes liegen kann, infolge der Beendigung der Therapie zu sterben.

Die Ethikerin argumentiert, dass es bei einem guten Einvernehmen zwischen Eltern und Ärzten möglich sein sollte, zu einem Konsens zu kommen. Falls das nicht möglich sei, müsse der Fall gerichtlich entschieden werden [5].

Eine Londoner Gruppe unter Andrew Bush betont in ihrer Publikation die Wichtigkeit der individuellen Entscheidung über eine Beatmung eines Kindes mit spinaler Muskelatrophie. In diesem Zusammenhang wird auch auf die Wichtigkeit einer interdisziplinären Diskussion und gemeinsamen Entscheidung hingewiesen.

Im Fall einer respiratorischen Insuffizienz wird zur Etablierung einer nächtlichen noninvasiven Beatmung, jedoch nicht $\mathrm{zu}$ einer Beatmung am Tag geraten. Die Autoren sehen in der Tagesbeatmung eine zu große Einschränkung des Kindes. Stattdessen wird vorgeschlagen, die Sekretmobilisation mittels eines „cough assist“, eines noninvasiven Geräts zur Unterstützung der inspiratorischen Atemarbeit und der forcierten Exspiration beim Abhusten, zu erleichtern.

Im Falle eines aufgrund einer akuten Verschlechterungssituation bereits intubierten und beatmeten Kindes schlägt der Autor vor, die Extubation und Umstellung auf noninvasive Beatmung vor. Eine Tracheostomie solle aber auf jeden Fall vermieden werden.

Wie auch die anderen Autoren betont Bush die hohe Wertigkeit der Kommunikation mit den Eltern und dem Betreuungsteam, um bei jeder Entscheidung einen möglichst breiten Konsens zu erzielen [6].

In einer Japanischen Arbeit eines Teams um Sakakihara wird eine Umfrage unter den Ärzten in 40 Spitälern zusammengefasst, in der an SMA 1 erkrankte Kinder beatmet werden. In 2/3 der Fälle hatten die Eltern explizit um Beginn der Beatmungstherapie gebeten und obwohl $80 \%$ der Ärzte fanden, dass die Le- 
bensqualität der beatmeten Kinder nicht adäquat war, meinte die Hälfte von ihnen, dass sie die Beatmung von sich aus einleiten würden, wenn sie die Betreuung eines Kindes mit SMA 1 übernähmen [7].

\section{Schlussfolgerung}

In dem vorliegenden Fall gab es keine Diskrepanz zwischen der Einstellung des Behandlungsteams und den Wünschen der Eltern. Dennoch muss überlegt werden, ob es gerechtfertigt ist, den Eltern in einem solchen Fall von ärztlicher Seite nahezulegen, eine maschinelle Beatmung ihres Kindes abzulehnen.

Eine Verlängerung des Lebens ihres Kindes wäre für die Eltern auf jeden Fall wünschenswert, da sie dadurch in der Lage wären, zumindest einige wenige - vorwiegend psychische - Entwicklungsschritte ihres Kindes mitzuerleben. Zudem kann ja nie mit Sicherheit feststehen, wie das Kind seine Lebensqualität selbst wahrnimmt und wie es für sich selbst entschieden hätte, wenn es dazu in der Lage gewesen wäre. Ein zumindest theoretisch möglicher wissenschaftlicher Fortschritt könnte auch zur Folge haben, dass in Zukunft eine Heilungschance für diese Krankheit bestünde, und die Lebensverlängerung bis zu diesem Zeitpunkt böte damit eine - wenn auch nur geringe Hoffnung auf vollständige Heilung.

Andererseits würde den Eltern im Falle einer maschinellen Beatmung, entweder zu Hause oder auch in einer medizinischen Einrichtung, das doch sehr wahrscheinliche Leiden des Kindes täglich vor Augen geführt werden. Nach der Entscheidung zur Einleitung einer Beatmung ist es verständlicherweise in der Folge auch ethisch wesentlich schwieriger, diese im Falle einer Reevaluation der Situation wieder zu beenden, da dies de facto das Absetzen einer ganz offensichtlich lebenserhaltenden Therapie erfordern würde.

Eine Lebensverlängerung ihres Kindes bedeutet für Eltern im Falle dieser schweren Erkrankung ohne wirklich realistische Chance auf Besserung oder gar Heilung eine doch sehr wahrscheinliche Leidensverlängerung. Die Kommunikation als ein wichtiger Aspekt der Eltern-Kind-Beziehung ist aller Wahrscheinlichkeit nach lebenslang unmöglich, was sowohl für die Eltern als auch für das Kind eine zusätzliche Belastung darstellt.

Diese Argumente haben zur Folge, dass sich die meisten Behandlungsteams im Gespräch mit den Eltern gegen die Etablierung einer maschinellen Beat- mung von Kindern mit spinaler Muskelatrophie Typ 1 entscheiden.

Ein ganz neuer Aspekt ergibt sich jedoch aufgrund einer Behandlungsoption, die wahrscheinlich in ganz naher Zukunft zur Verfügung stehen wird. Diese besteht in einer medikamentösen Therapie, die auf genetischer Ebene in die Exprimierung des defekten Exons eingreift. Diese Therapie befindet sich zurzeit in der klinischen Studienphase und steht nicht für alle Betroffenen zur Verfügung. Sie scheint nach ersten Ergebnissen zwar die Funktion der Skelettmuskulatur zu verbessern, die Atemleistung jedoch nicht wesentlich zu beeinflussen.

Funding Open access funding provided by Paracelsus Medical University.

Interessenkonflikt M. Radner und A. Henry geben an, dass kein Interessenkonflikt besteht.

Open Access Dieser Artikel wird unter der Creative Commons Namensnennung 4.0 International Lizenz (http:// creativecommons.org/licenses/by/4.0/deed.de) veröffentlicht, welche die Nutzung, Vervielfältigung, Bearbeitung, Verbreitung und Wiedergabe in jeglichem Medium und Format erlaubt, sofern Sie den/die ursprünglichen Autor(en) und die Quelle ordnungsgemäß nennen, einen Link zur Creative Commons Lizenz beifügen und angeben, ob Änderungen vorgenommen wurden.

\section{Literatur}

1. Farrar MA, Vucic S, Johnston HM, et al. Pathophysiological insights derived by natural history and motor function of spinal muscular atrophy. J Pediatr. 2013;162:155-9.

2. Gray K, Isaacs D, Kilham HA, et al. Spinal muscular atrophy type I: do the benefits of ventilation compensate for its burdens? J Paediatr Child Health. 2013;49:807-12.

3. Jäger G. Ethische Problemsituationen in der Pädiatrie: Modell und Patientenbeispiel für eine Entscheidungsfindung im Behandlungsteam. Paediatrica. 2013;24(2):7-9.

4. Lemoine TJ, Swoboda KJ, Bratton SL, et al. Spinal muscular atrophy type 1: are proactive respiratory interventions associated with longer survival? Pediatr Crit Care Med. 2012;13(3):e161-e5.

5. Ryan M, Kilham H, Jacobe St, et al. Spinal muscular atrophy type 1: islong tern mechanicalventilation ethical? JPaediatr Child Health. 2007;43:237-42.

6. Bush A, Fraser J, Patron J, et al. Respiratory management of the infant with type 1 spinal muscular atrophy. Arch Dis Child. 2005;90:709-11.

7. Sakakira Y, Kubota M, Sim S, et al. Long-term ventilator support in patients with Werdnig-Hoffmann disease. Pediatr Int. 2000;42(4):359-63. 